\title{
El impacto de la nutrición en las calificaciones escolares a nivel primaria: un estudio del Programa de Desayunos Escolares de la Ciudad de México
}

\author{
Impact of nutrition on scores at elementary schools: \\ A study of the School Breakfast Program in Mexico City \\ Armando Sánchez Vargas * 1, Anadeli Naranjo Carbajal, Isalia Nava Bolaños \\ Universidad Nacional Autónoma de México, México
}

Recibido el 26 de octubre de 2018; aceptado el 7 de enero de 2019

Disponible en Internet el: 9 de enero de 2019

\section{Resumen}

Este artículo investiga el efecto de la participación en el Programa de Desayunos Escolares de la Ciudad de México sobre las calificaciones de niñas y niños de primaria. Los datos provienen del levantamiento de encuestas para la evaluación de impacto del programa, las cuales contienen información de estudiantes de cuarto y quinto grado de 17 escuelas públicas de la ciudad para el ciclo escolar 2017-2018. El estudio del impacto tiene un enfoque comparativo entre alumnos de escuelas beneficiarias y no beneficiarias. Los resultados de las estimaciones sugieren que el programa de alimentación escolar no propicia cambios significativos en las calificaciones, lo cual contrasta con hallazgos en otros países donde sí se ha encontrado un efecto significativo. Los hallazgos permiten además identificar la existencia de una relación inversa entre las calificaciones y las faltas a clases, así como de una relación directa con los antecedentes educativos de la familia y la disponibilidad adicional de otros programas sociales.

Código JEL: I18, I28, H51, H52

Palabras clave: Desempeño escolar; Nutrición; Programa de desayunos escolares

\footnotetext{
* Autor para correspondencia

Correo electrónico armando_sanchez123@hotmail.com (A. Sánchez Vargas).

La revisión por pares es responsabilidad de la Universidad Nacional Autónoma de México. 


\begin{abstract}
This paper investigates the effect of participation in the School Breakfast Program of Mexico City on children's achievements. We use data from a survey to assess the impact of such program on 4th and 5th grade students of 17 elementary public schools in the city for the 2017-2018 school year. The study has a comparative approach between students of beneficiary and non-beneficiary schools. Controlling for other factors, the results of the estimates suggest that the program does not promote significant changes on scores, which contrasts with findings in other countries where a significant effect has been found. Our results also suggest the existence of an inverse relationship between scores and absenteeism, and a positive relationship with the family's educational background and the availability of other social programs.
\end{abstract}

JEl code: $\mathrm{I} 18, \mathrm{I} 28, \mathrm{H} 51, \mathrm{H} 52$

Keywords: School performance; Nutrition; School breakfast program

\title{
Introducción
}

Existe evidencia empírica robusta a nivel internacional que sugiere que las niñas y niños con mayor acceso a una alimentación nutritiva tienden a presentar un mejor rendimiento escolar -en sus calificaciones-, en comparación con aquellos en situación de malnutrición. De hecho, una dieta rica en vitaminas y minerales podría mejorar la retención y la memoria de los estudiantes (World Food Program, 2017; Frisvold, 2015). Así, los niños en situación de pobreza suelen ser los más propensos ante las diferentes formas de malnutrición (Federal Interagency Forum on Child and Family Statistics, 2007), y por lo tanto, presentan mayor riesgo de ver afectado su desarrollo físico y cognitivo a una edad temprana.

Más aún, se sabe que los problemas de nutrición como el sobrepeso y la obesidad tienen impacto sobre el desarrollo económico y la formación de capital humano de los países (Torres y Rojas, 2018). Ante tales circunstancias, los programas sociales de asistencia alimentaria juegan un papel crucial en la reducción de las brechas de nutrición, desempeño escolar y desarrollo infantil.

En este contexto, en la Ciudad de México, el Programa de Desayunos Escolares busca contribuir a la mejora en la alimentación de la población en edad escolar, otorgando desayunos fríos a niñas y niños inscritos en escuelas públicas de nivel básico y preferentemente entre aquellos de escasos recursos (Gaceta Oficial de la Ciudad de México, 2018). El cambio en los hábitos alimenticios que fomenta dicho programa podría incidir no sólo en la nutrición, sino también sobre los resultados académicos de los participantes. A pesar de los beneficios que otorga, este programa mexicano de alimentación escolar, de amplia cobertura y gran tra- 
yectoria, no ha sido objeto de una evaluación cuantitativa en términos de su incidencia sobre las calificaciones de las niñas y niños que consumen las raciones alimentarias.

Así, el objetivo de este artículo es analizar por primera vez la posible existencia de una relación causal entre la participación en dicho programa y los resultados en las calificaciones de las niñas y niños que consumen el desayuno. Es decir, se busca averiguar si el programa tiene otros efectos positivos colaterales en las niñas y niños participantes, además de los impactos nutricionales que busca el programa. Los datos empleados provienen de una nueva encuesta que se levantó para la evaluación de los impactos del programa en escuelas de la Ciudad de México, la cual permite analizar a estudiantes de cuarto y quinto grado de primaria de 17 escuelas, con información para el periodo agosto-diciembre del ciclo escolar 20172018. El estudio tiene un enfoque de comparación entre estudiantes de escuelas participantes y no participantes.

El modelo teórico subyacente consiste en una función de producción de los logros escolares -calificaciones- que contempla ampliamente la incidencia de la nutrición. Las estimaciones son realizadas con modelos de regresión múltiple y modelos probit ordenados. Los resultados sugieren que la participación en el Programa de Desayunos Escolares no muestra una relación significativa con los promedios escolares de los estudiantes, lo que podría implicar que el programa sólo contribuye con la nutrición y no genera efectos adicionales en el desempeño escolar, como sucede en programas similares de otros países. Adicionalmente, se encontró que las calificaciones de las niños y niños están asociadas de manera inversa con las faltas escolares y de manera directa con variables como el nivel de educación de los padres y con la disponibilidad de otros programas sociales que podrían ser complementarios al programa de desayunos escolares.

El documento está organizado en cuatro secciones, más esta introducción y las conclusiones. En la primera sección se presentan los antecedentes del programa, así como los principales hallazgos de la bibliografía previa. En la segunda sección se realiza una descripción de los datos colectados, así como de los principales estadísticos. En la tercera sección se desarrolla el marco teórico y métodos de estimación empleados para la investigación. En la cuarta sección se discuten los resultados. 


\section{Antecedentes}

El Programa de Desayuno Escolares de la Ciudad de México fue instaurado en 1942, teniendo como antecedente a organizaciones altruistas, quienes brindaban raciones alimentarias a niñas y niños en condiciones de pobreza. Es hasta 1997 cuando, por decreto del Diario Oficial de la Federación, se establece la descentralización del programa y el alcance nacional del mismo. Con dicha reforma se transformó también el contenido nutricional de los desayunos. Desde entonces, su composición ha evolucionado hacia un mayor apego a los lineamientos y normas federales, los cuales buscan cumplir con los nutrimientos requeridos para el sano desarrollo de los menores en edad escolar (Gaceta Oficial de la Ciudad de México, 2018).

De acuerdo con los Lineamientos de la Estrategia Integral de Asistencia Social Alimentaria (EIASA, 2017) del Sistema Nacional para el Desarrollo Integral de la Familia (SNDIF), cada desayuno aporta 360 kilocalorías totales, lo cual representa 25 por ciento del contenido energético diario recomendado para esta etapa de crecimiento. Específicamente, el desayuno aporta 5.0 gramos de azúcares añadida, 5.4 gramos de fibra, 360 miligramos de sodio, un máximo de 60 por ciento de kilocalorías de hidratos de carbono, 15 por ciento de kilocalorías de proteína, 25 por ciento de kilocalorías de grasas totales y 10 por ciento de kilocalorías de grasa saturada.

En 2017 el programa atendió a 686,661 estudiantes de escuelas públicas de educación básica, especial e inicial de las 16 delegaciones de la ciudad, dando prioridad a la población de escasos recursos y de los territorios con un índice de desarrollo social muy bajo, bajo y medio. Donde, dicho indicador representa una medida de necesidades básicas insatisfechas, ${ }^{1}$ que permite comparar las condiciones de desarrollo social de la población de diferentes territorios (Gaceta Oficial de la Ciudad de México, 2016).

Estudios previos han evaluado la incidencia de los programas de alimentación escolar implementados en diversos países sobre dos tipos de resultados: indicadores de nutrición e indicadores de logros escolares. Este artículo se enfocará en el estudio del rendimiento escolar. $\mathrm{Al}$ respecto, Frisvold (2015) examina el impacto del Programa de Desayunos Escolares en Estados Unidos de América mediante los métodos de diferencias en diferencias y regresión discontinua, aprovechando mandatos estatales para comparar escuelas con alto y bajo nivel de estudiantes elegibles. Los resultados señalan que la disponibilidad del desayuno contribuye a mejorar las calificaciones en matemáticas y lectura. Meyers et al. (1989) examinan este

\footnotetext{
${ }^{1}$ Los indicadores de necesidades insatisfechas que se utilizan en el cálculo del índice de desarrollo social de la Ciudad de México son: 1) indicador de calidad y espacio disponible en la vivienda; 2) indicador de acceso a electricidad; 3 ) indicador de bienes durables; 4) indicador de adecuación sanitaria; 5) indicador de acceso a seguridad social y servicio médico; 6) indicador de rezago educativo. Al combinar estos indicadores mediante una media aritmética ponderada se clasifican las áreas geográficas en cuatro rubros. Dónde dicho índice va de 0 a 1 y 1 representa el de mayor desarrollo. De acuerdo con esta clasificación existen 4 categorías: muy bajo (menor a 0.7 ), bajo (entre 0.7 y 0.8 ), medio (entre 0.8 y 0.9 ) y alto (mayor a 0.9 ).
} 
mismo programa americano, comparando a estudiantes que participaron por primera vez en el programa con aquellos elegibles, pero no participantes. Los hallazgos muestran que los niños beneficiarios mejoraron significativamente sus calificaciones en pruebas estandarizadas.

Anzman-Frasca et al. (2015) estiman el impacto del programa estadounidense de desayunos escolares, pero en su modalidad de entrega en el salón de clases. Su diseño es cuasi experimental y usan pesos de puntaje de propensión para ajustar por características no observables. Los autores concluyen que las calificaciones escolares no mostraron diferencias estadísticamente significativas entre tratados y no tratados. En la misma dirección, Imberman y Kugler (2014) estudian también la modalidad de desayuno dentro del salón. A diferencia de los autores previos, emplean una metodología de diferencias en diferencias y encuentran que las puntuaciones de matemáticas incrementan en 0.09 desviaciones estándar y las de lectura en 0.06 desviaciones estándar cuando los estudiantes pertenecen a la nueva modalidad.

Otra evidencia para Estados Unidos de América, es la evaluación al Programa Nacional de Almuerzos Escolares (Hinrich, 2010), la cual mostró impactos de largo plazo sobre los resultados educativos de los beneficiarios. La evaluación usa variables instrumentales y contempla un cambio de mandato en la composición del almuerzo, así como la disponibilidad de cohortes de individuos con exposición a los dos tipos de desayunos. La evidencia señala que el programa incide positivamente sobre los años de escolaridad. Un aumentando de $10 \%$ en la exposición al almuerzo con el nuevo cambio de modalidad aumenta 0.36 años de escolaridad en las mujeres y en casi un año en los hombres.

Vermeersch y Kremer (2004) evalúan una intervención que consiste en subsidios para comidas a escolares de la región occidental de Kenya con un enfoque comparativo entre escuelas participantes y no participantes. Sus hallazgos señalan que los estudiantes tratados, y en escuelas donde los profesores cuentan con más años de experiencia, mejoraron sus calificaciones en 0.38 desviaciones estándar. Belot y James (2011) usan la modificación en una legislación de 2004 en Reino Unido que cambia de manera radical la composición de las comidas, en escuelas del poblado de Greenwich, para identificar el impacto causal del programa. Mediante un enfoque de diferencias en diferencias, los autores encuentran un aumento en la proporción de niños que alcanzan una mejor calificación en matemáticas, en inglés y en ciencia.

Afridi (2011) estudia los efectos del cambio de mandato de un programa de alimentación sobre la participación escolar en el área rural de India. El mandato implementa la transición de un esquema que brinda granos y alimentos para llevar a casa hacia un esquema donde se ofrece comida preparada a los niños directamente en sus escuelas. Mediante una metodología de diferencias en diferencias, el autor encuentra que la reorientación del programa tuvo un efecto significativo sobre las tasas de participación diaria de estudiantes de los primeros grados escolares, especialmente los resultados muestran efectos significativos para el caso de las niñas. 
Un estudio para Filipinas indica que una mejor nutrición en la infancia temprana, producto de la intervención de un programa de alimentación infantil, tiene un impacto positivo sobre los resultados escolares de los menores (Glewwe et al., 2001). Específicamente, los autores encuentran que la inscripción escolar aumenta y la repetición de grado disminuye y concluyen que cada dólar de inversión en un programa de alimentación a infantes puede proporcionar ganancias de hasta 3 dólares en desempeño académico para estos menores.

Aunque, la evidencia para Chile muestra que el programa de Desayunos Escolares implementado en este país tiene efectos nulos sobre los resultados de logros escolares (McEwan, P., 2013). En dicho estudio fueron evaluados mediante regresión discontinua los indicadores de inscripción escolar, repetición de año, asistencia y calificaciones. Los resultados muestran que ninguno de estos indicadores presentó cambios significativos como producto de la intervención. El autor atribuye dicho hallazgo al alto contenido calórico de los desayunos y poco contenido realmente nutricional.

En este contexto, cabe destacar que el programa de Desayunos Escolares de la Ciudad de México, nunca ha sido evaluado en términos de su impacto en el rendimiento escolar de las niñas y niños participantes. Tampoco se ha estudiado la incidencia del desayuno escolar en los logros académicos, mediante una encuesta de una población de estudiantes con un diseño comparativo que contemple grupos de tratamiento y control. Por lo anterior, el principal aporte de esta investigación consiste en realizar un primer estudio estadístico, que arroja evidencia empírica causal confiable, basada en una encuesta novedosa sobre el impacto de la participación en el programa y los resultados de promedio escolar de las niñas y niños que reciben el beneficio.

\section{Datos, encuesta y estadística descriptiva}

La base de datos proviene de un levantamiento de 1,112 cuestionarios a madres y padres de familia, para evaluar el impacto del Programa de Desayunos Escolares de la Ciudad de México, a estos datos se agregaron además los registros administrativos del programa. ${ }^{2}$ Los datos fueron recolectados durante los meses de noviembre y diciembre de 2017 y proporcionan información del ciclo escolar 2017-2018. La población objetivo fueron los estudiantes de

\footnotetext{
${ }^{2}$ Adicionalmente se imputó a la base de datos información sobre servicios y calidad de las viviendas con base en la Encuesta Nacional de Ingresos y Gastos de los Hogares 2016 (ENIGH, 2016), información del tamaño e infraestructura de las escuelas a partir de datos de la Secretaria de Educación Pública (SEP, 2015), e información de precios de alimentos básicos por región geográfica. Los datos de la ENIGH y de precios fueron asignados por medio del software ArcGis con precisión a nivel de Área Geoestadística Básica (AGEB). Los datos de SEP fueron atribuidos por medio de la clave de centro de trabajo.
} 
cuarto y quinto grado de 17 escuelas primarias de los turnos matutino y continuo, ubicados en ocho delegaciones de la Ciudad de México, ${ }^{3}$ tal como lo muestra la figura 1.

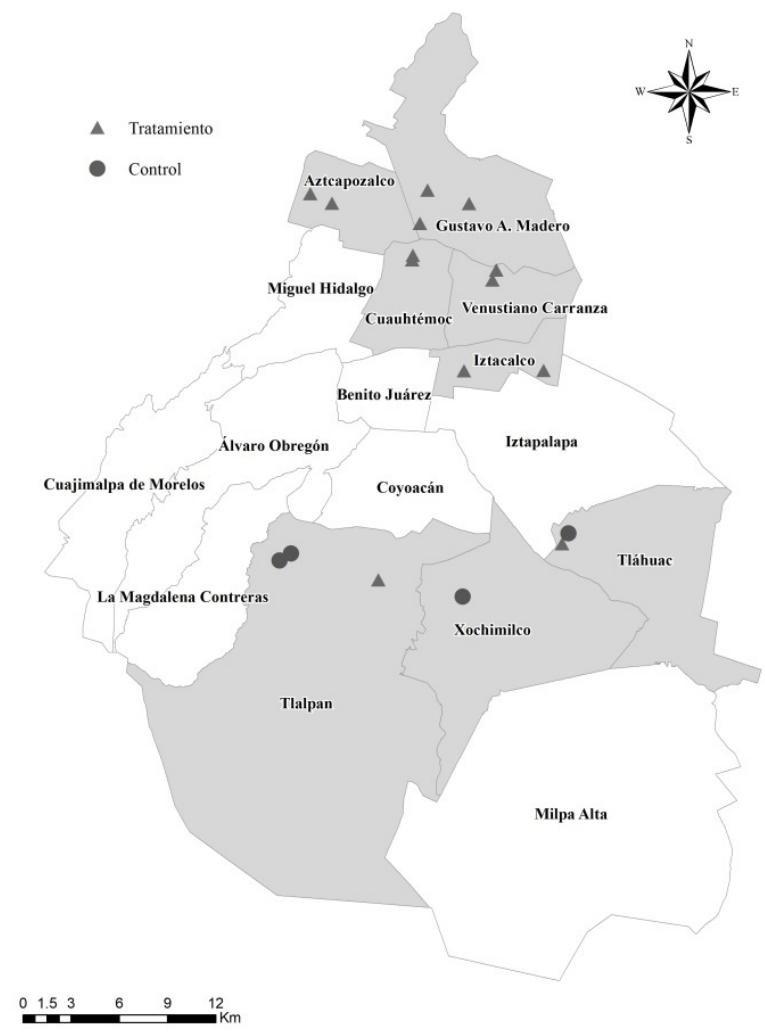

Figura 1. Distribución de la población evaluada.

Fuente: Elaboración propia con base en Encuesta levantada para la Evaluación del Impacto del Programa de Desayunos Escolares de la Ciudad de México (2017).

La elección de las escuelas participantes en el estudio estuvo restringida por las condiciones de infraestructura o disponibilidad como consecuencia del sismo ocurrido el 19 de septiembre de 2017 en la Ciudad de México. Aquellas escuelas con las condiciones adecuadas fueron

\footnotetext{
${ }^{3}$ Adicionalmente se llevó a cabo una prueba piloto en una escuela primaria de la delegación Miguel Hidalgo.
} 
invitadas a participar y de éstas un grupo de 17 escuelas se autopropuso para colaborar con el estudio, en consecuencia, los resultados estadísticos solo son aplicables a la población de esas escuelas y no es posible extender los resultados al total de escuelas de la ciudad.

De las 17 escuelas que participaron, 13 son beneficiarias del programa y 4 son escuelas no beneficiarias. Todas las niñas y niños inscritos en los niveles de cuarto y quinto grado de los planteles seleccionados fueron considerados para el estudio. Las solicitudes formales a las escuelas participantes fueron enviadas con anticipación, así mismo, los directivos enviaron comunicados a los padres de familia para informar sobre la evaluación, por tanto, se excluyeron a los estudiantes cuyos padres rechazaron el consentimiento.

Del total de alumnos inscritos en cuarto y quinto grado de las 17 escuelas primarias participantes, se obtuvieron 1,112 cuestionarios completos. Sin embargo, se detectó que no todos los beneficiarios consumían el desayuno. Por lo tanto, a partir de las preguntas a tutores se identificó a los estudiantes participantes que verdaderamente ingirieron los alimentos del programa, lo cual asegura que se evalué de manera apropiada los impactos para quienes realmente están expuestos a los beneficios de la intervención. La población final está conformada por 1,051 estudiantes, de los cuales, 749 (71.3\%) pertenecen al grupo de tratamiento y 302 $(28.7 \%)$ al grupo de control (ver figura 2).

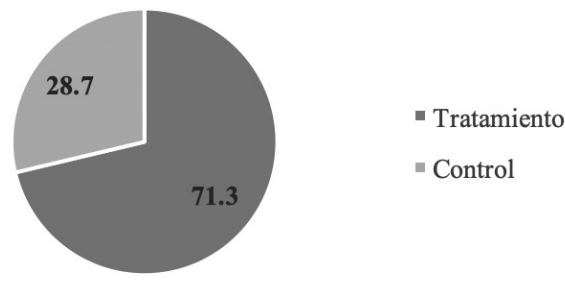

Figura 2. Distribución de estudiantes según grupos de tratamiento y control (agosto-diciembre, 2017)

Fuente: Elaboración propia con base en Encuesta levantada para la Evaluación del Impacto del Programa de Desayunos Escolares de la Ciudad de México (2017).

En términos del indicador de rendimiento académico, éste es medido como la calificación general de las niñas y niños en el período agosto-diciembre del ciclo escolar 2017-2018. Dicho indicador se conforma por intervalos, dónde: 1 es una calificación menor a 6; 2 indica entre 6.0 y $6.5 ; 3$ equivale a un rango de entre 6.6 y $7.0 ; 4$ representa entre 7.1 y $7.5 ; 5$ entre 7.6 y $8.0 ; 6$ se refiere a puntajes entre 8.1 y $8.5 ; 7$ entre 8.6 y $9.0 ; 8$ entre 9.1 y 9.5 ; y 9 es entre 9.6 y 10. En promedio, los estudiantes de ambos grupos obtuvieron notas de entre 8.6 y 9.0. 
Si se comparan las calificaciones promedio del grupo de control con el grupo de tratamiento, sin controlar por alguna otra variable, se obtiene que los alumnos inscritos en escuelas de control que no reciben el desayuno presentan en mayor porcentaje promedios más altos (superiores a 9.0), en comparación con su contraparte de tratamiento (ver figura 3). Sin embargo, estos resultados no reflejan aún el impacto causal del programa sobre los resultados escolares, debido a que no se controla por características observables y no observables, como en un modelo de regresión.

Dado que la participación en el programa no fue asignada de manera aleatoria, los estudiantes participantes y no participantes podrían presentar diferentes perfiles. La presencia de determinadas características podría predecir tanto la probabilidad de participar en el programa, como la de obtener calificaciones más favorables lo cual puede sesgar los resultados estadísticos. Por ejemplo, el nivel de ingresos del hogar o la escolaridad de los padres podrían incidir en el desempeño escolar de las hijas e hijos, estas características se deberían tomar en cuanta en los modelos para estimar los impactos causales.

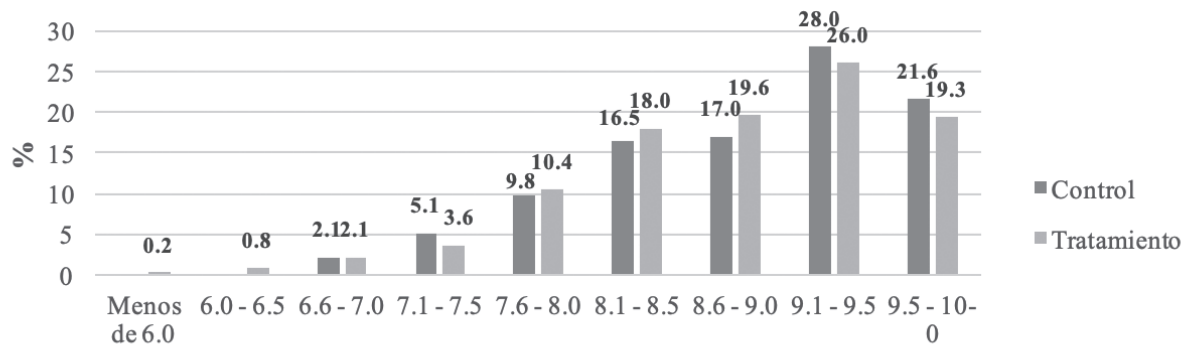

Promedio escolar

Figura 3. Promedio escolar según grupos de tratamiento y control (agosto-diciembre, 2017)

Fuente: Elaboración propia con base en Encuesta levantada para la Evaluación del Impacto del Programa de Desayunos Escolares de la Ciudad de México (2017).

Con la finalidad de controlar por la mayor parte de los factores que explican las calificaciones y siguiendo a Behrman y Hoddinot (2001) y a Glewwe (2005) se realizan pruebas de diferencias de medias, incluyendo diferentes grupos de regresores: características de los estudiantes, factores del hogar, particularidades de las escuelas, disponibilidad de programas sociales, precios de alimentos básicos y ubicación geográfica. El resumen estadístico de dichas características es presentado en la tabla 1. La primera columna presenta la media y desviación estándar de las características de la población. Las columnas dos y tres muestran 
dichos estadísticos de manera desglosada para los estudiantes beneficiarios y no beneficiarios, respectivamente. Las columnas cuatro, cinco y seis ofrecen evidencia de la diferencia estadística entre los resultados de ambos grupos. Específicamente, la cuarta columna presenta una prueba de medias simple, mientras que, las columnas cinco y seis contemplan además los efectos fijos por escuela y por escuela y delegación.

La distribución de la población según sexo es equitativa, $50.3 \%$ son niñas y $49.7 \%$ niños. La población de estudiantes registró un promedio de 118 meses de edad (9.8 años). Se encontró que $47.9 \%$ de los alumnos faltaron a clases, al menos una ocasión en el mes anterior al levantamiento de los datos. El ingreso mensual promedio de los hogares en que habitan estas niñas y niños fue de 6,024.4 pesos. El $95.7 \%$ de los infantes viven en el mismo hogar que su mamá, mientras que, en el caso del papá este porcentaje es del 69.2\%.

Las madres de estos hogares que participan en el mercado laboral representan 68.9\%, mientras que en los padres dicha cifra asciende al 93.7\%. En relación con la escolaridad de las madres, $47.1 \%$ cuenta con un nivel de estudios de bachillerato y únicamente $17.9 \%$ de ellas tienen licenciatura o un grado mayor. Por su parte, los padres con bachillerato son $42.8 \%$ y con licenciatura o nivel mayor $15.6 \%$. En cuanto a la calidad de las viviendas de la población, éstas cuentan en promedio con nueve focos.

De los estudiantes evaluados, 5.4\% son beneficiarios del Programa de Becas Escolares para Niñas y Niños en Condición de Vulnerabilidad Social de la Ciudad de México. Además, 13.7\% pertenecen al Programa de Niños Talento de esta ciudad. Las escuelas a las que asisten los estudiantes evaluados se componen en promedio de 32 niños por cada profesor. Los directivos de dichos planteles tienen en promedio nueve años de experiencia en el área dirigente.

A partir del análisis de las diferencias de medias simples y condicionadas, podemos señalar que no existen diferencias significativas entre ambos grupos, en términos de la gran mayoría de las características descritas. Por lo cual, los grupos de control y tratamiento podrían ser comparables. Sin embargo, algunos de estos factores señalan diferencia en la media de los dos grupos bajo ciertas especificaciones, por lo cual es importante controlar en las estimaciones por dichas variables.

Específicamente, la variable del hogar referente a la disponibilidad de focos en la vivienda muestra una diferencia significativa bajo las tres especificaciones empleadas. Lo anterior podría indicar que existen disparidades en la calidad de las viviendas, de modo que, el número de focos es superior en las de control (11) en relación con las de tratamiento (8). Por su parte, la probabilidad de inasistencia presenta una diferencia en las pruebas de la cuarta y sexta columna, indicando que los alumnos beneficiarios podrían presentar mayor probabilidad de faltar a clases (50.8\%), en comparación con los no beneficiarios (40.8\%).

El empleo de la madre mostró diferencias en las especificaciones de medias simples y con efectos fijos por escuela, sin embargo, ésta variación desapareció cuando se controlaron 
los efectos por delegación en el modelo de regresión. Finalmente, la variable de tamaño de grupo señala que en pruebas de medias simples podrían existir diferencias entre los grupos de control y tratamiento en términos de esta variable. De modo que, el promedio de alumnos por profesor es de 30 en las escuelas beneficiarias y de 36 en las no beneficiarias.

Tabla 1

Descripción estadística de las características de la población.

\begin{tabular}{|c|c|c|c|c|c|c|}
\hline Variables & $\begin{array}{l}\text { Total } \\
\text { (1) }\end{array}$ & $\begin{array}{c}\text { Tratamiento } \\
\text { (2) }\end{array}$ & $\begin{array}{c}\text { Control } \\
\text { (3) }\end{array}$ & $\begin{array}{c}\text { P-Value } \\
\text { (4) }\end{array}$ & $\begin{array}{l}\text { P-Value con } \\
\text { efectos fijos } \\
\text { por escuela } \\
\text { (5) }\end{array}$ & $\begin{array}{c}\text { P-Value con } \\
\text { efectos fijos } \\
\text { por escuela } \\
\text { y delegación } \\
\text { (6) }\end{array}$ \\
\hline Sexo (niña) & $\begin{array}{l}0.503 \\
(0.500)\end{array}$ & $\begin{array}{c}0.505 \\
(0.500)\end{array}$ & $\begin{array}{c}0.500 \\
(0.500)\end{array}$ & 0.891 & 0.987 & 0.922 \\
\hline Edad en meses & $\begin{array}{l}118.414 \\
(7.126)\end{array}$ & $\begin{array}{l}118.179 \\
(7.259)\end{array}$ & $\begin{array}{l}118.997 \\
(6.761)\end{array}$ & 0.092 & 0.842 & 0.166 \\
\hline $\begin{array}{l}\text { Probabilidad de } \\
\text { inasistencia }\end{array}$ & $\begin{array}{l}0.479 \\
(0.499)\end{array}$ & $\begin{array}{c}0.508 \\
(0.500)\end{array}$ & $\begin{array}{l}0.400 \\
(0.490)\end{array}$ & 0.005 & 1.000 & 0.042 \\
\hline Ingreso & $\begin{array}{c}6024.391 \\
(3681.853)\end{array}$ & $\begin{array}{l}6128.022 \\
(3776.471)\end{array}$ & $\begin{array}{l}5773.787 \\
(3436.323)\end{array}$ & 0.190 & 0.000 & 0.919 \\
\hline Logaritmo de ingreso & $\begin{array}{r}8.553 \\
(0.539)\end{array}$ & $\begin{array}{l}8.567 \\
(0.545)\end{array}$ & $\begin{array}{r}8.520 \\
(0.525)\end{array}$ & 0.225 & 0.000 & 0.998 \\
\hline $\begin{array}{l}\text { Habita la madre en } \\
\text { casa }\end{array}$ & $\begin{array}{l}0.957 \\
(0.203)\end{array}$ & $\begin{array}{c}0.958 \\
(0.200)\end{array}$ & $\begin{array}{c}0.953 \\
(0.212)\end{array}$ & 0.700 & 0.763 & 0.217 \\
\hline $\begin{array}{l}\text { Habita el padre en } \\
\text { casa }\end{array}$ & $\begin{array}{c}0.692 \\
(0.461)\end{array}$ & $\begin{array}{c}0.681 \\
(0.466)\end{array}$ & $\begin{array}{c}0.718 \\
(0.450)\end{array}$ & 0.245 & 0.889 & 0.968 \\
\hline Trabaja la madre & $\begin{array}{c}0.740 \\
(0.438)\end{array}$ & $\begin{array}{c}0.760 \\
(0.427)\end{array}$ & $\begin{array}{c}0.689 \\
(0.463)\end{array}$ & 0.020 & 0.002 & 0.102 \\
\hline Trabaja el padre & $\begin{array}{c}0.950 \\
(0.217)\end{array}$ & $\begin{array}{l}0.956 \\
(0.206)\end{array}$ & $\begin{array}{l}0.937 \\
(0.243)\end{array}$ & 0.267 & 0.596 & 0.586 \\
\hline $\begin{array}{l}\text { Educación de la ma- } \\
\text { dre: preparatoria }\end{array}$ & $\begin{array}{c}0.468 \\
(0.499)\end{array}$ & $\begin{array}{c}0.467 \\
(0.499)\end{array}$ & $\begin{array}{c}0.471 \\
(0.500)\end{array}$ & 0.914 & 0.558 & 0.511 \\
\hline Educación de la & 0.176 & 0.174 & 0.179 & & & \\
\hline $\begin{array}{l}\text { madre: licenciatura o } \\
\text { grado mayor }\end{array}$ & $(0.380)$ & $(0.379)$ & $(0.383)$ & 0.872 & 0.079 & 0.840 \\
\hline
\end{tabular}




\begin{tabular}{|c|c|c|c|c|c|c|}
\hline Variables & $\begin{array}{l}\text { Total } \\
(1)\end{array}$ & $\begin{array}{c}\text { Tratamiento } \\
\text { (2) }\end{array}$ & $\begin{array}{l}\text { Control } \\
\text { (3) }\end{array}$ & $\begin{array}{l}\text { P-Value } \\
\text { (4) }\end{array}$ & $\begin{array}{c}\text { P-Value con } \\
\text { efectos fijos } \\
\text { por escuela } \\
\text { (5) }\end{array}$ & $\begin{array}{c}\text { P-Value con } \\
\text { efectos fijos } \\
\text { por escuela } \\
\text { y delegación } \\
\text { (6) }\end{array}$ \\
\hline $\begin{array}{l}\text { Educación del pa- } \\
\text { dre: preparatoria }\end{array}$ & $\begin{array}{c}0.396 \\
(0.489)\end{array}$ & $\begin{array}{c}0.383 \\
(0.486)\end{array}$ & $\begin{array}{c}0.428 \\
(0.495)\end{array}$ & 0.228 & 0.857 & 0.960 \\
\hline $\begin{array}{l}\text { Educación del pa- } \\
\text { dre: licenciatura o } \\
\text { grado mayor }\end{array}$ & $\begin{array}{l}0.203 \\
(0.402)\end{array}$ & $\begin{array}{l}0.222 \\
(0.415)\end{array}$ & $\begin{array}{l}0.156 \\
(0.363)\end{array}$ & 0.032 & 0.140 & 0.916 \\
\hline $\begin{array}{l}\text { Número de focos } \\
\text { de la vivienda }\end{array}$ & $\begin{array}{l}9.024 \\
(4.769)\end{array}$ & $\begin{array}{l}8.160 \\
(2.617)\end{array}$ & $\begin{array}{l}11.167 \\
(7.474)\end{array}$ & 0.000 & 0.000 & 0.000 \\
\hline $\begin{array}{l}\text { Programa de Becas } \\
\text { Escolares }\end{array}$ & $\begin{array}{c}0.054 \\
(0.226)\end{array}$ & $\begin{array}{c}0.056 \\
(0.230)\end{array}$ & $\begin{array}{c}0.050 \\
(0.217)\end{array}$ & 0.679 & 0.640 & 0.963 \\
\hline $\begin{array}{l}\text { Programa Niños } \\
\text { Talento }\end{array}$ & $\begin{array}{c}0.137 \\
(0.344)\end{array}$ & $\begin{array}{c}0.136 \\
(0.343)\end{array}$ & $\begin{array}{c}0.139 \\
(0.346)\end{array}$ & 0.902 & 0.124 & 0.152 \\
\hline $\begin{array}{l}\text { Relación alumnos } \\
\text { por profesor }\end{array}$ & $\begin{array}{l}31.565 \\
(3.782)\end{array}$ & $\begin{array}{l}29.893 \\
(2.602)\end{array}$ & $\begin{array}{l}35.713 \\
(2.976)\end{array}$ & 0.000 & - & - \\
\hline $\begin{array}{l}\text { Experiencia del di- } \\
\text { rector }^{\mathrm{a}}\end{array}$ & $\begin{array}{l}9.313 \\
(6.156)\end{array}$ & $\begin{array}{l}9.286 \\
(6.952)\end{array}$ & $\begin{array}{l}9.394 \\
(2.684)\end{array}$ & 0.809 & - & - \\
\hline
\end{tabular}

Fuente: Elaboración propia con base en Encuesta levantada para la Evaluación del Impacto del Programa de Desayunos Escolares de la Ciudad de México (2017).

a Las variables de alumnos por profesor y experiencia del director se encuentran a nivel de escuela, por lo cual no es posible aplicar efectos fijos.

\section{Marco analítico: función de producción de logros académicos}

El marco analítico está basado en una función de producción de logros académicos -calificaciones- que considera ampliamente la influencia de variables asociadas a la nutrición de las niñas y niños (Glewwe, 2005). Dicho modelo establece que los logros escolares están determinados no solo por factores individuales, familiares y de mercado en el periodo actual, sino también por la influencia de éstos en periodos anteriores. De hecho, el modelo considera distintas variables en tres periodos de tiempo como determinantes de los logros escolares.

El primer periodo comprende desde la etapa de concepción hasta los 24 meses después del nacimiento. La segunda etapa va de los 24 meses a los 6 años. El tercer período comprende de los 6 a los 11 años, tiempo en que, por lo general, los estudiantes se encuentran en 
la educación primaria. Lo anterior permite estudiar de manera dinámica la relación entre la nutrición presente y pasada y los logros académicos, como se muestra en la ecuación 1. La variable de resultado en este caso se refiere a las calificaciones que obtuvieron los estudiantes en el tercer periodo (en el de edad escolar) y se representa por la variable .

$$
L_{3}=L\left(N_{1}, N_{2}, N_{3}, R E_{1}, R E_{2}, R E_{3}, \gamma, E, A E\right)
$$

Dónde: $N_{t}$ es la nutrición del estudiante en el período ; son los insumos escolares proporcionados por los padres de familia en el período (libros, materiales educativos y tiempo dedicado al apoyo de las actividades escolares); son las habilidades cognitivas innatas del estudiante; son las características de la escuela; son los años de escolaridad.

Para fines de simplificación, generalmente se consideran constantes los insumos escolares, las características de la escuela, la habilidad innata del menor y los años de escolaridad. Lo cual permite determinar cómo los cambios en las condiciones de nutrición en los tres períodos mencionados tendrán efectos sobre las calificaciones de los escolares. Los insumos educativos ( $A E$ ) y los años de escolaridad $\left(R E_{1}, R E_{2}, R E_{3}\right)$ se encuentran en gran medida bajo la decisión de los padres y reflejan su preferencias sobre la educación de sus hijos. Al mismo tiempo, la asignación de las familias para comprar insumos educativos también refleja las preferencias de los tutores en términos de nutrición de los menores, lo anterior debido a que deben decidir la mayor o menor asignación entre ambos tipos de bienes.

Dichas variables bajo control total o parcial de los padres podrían ser consideradas como endógenas. Una forma de enfrentar la endogeneidad de dichas variables es planteando una forma reducida de la función de producción de habilidades escolares, donde, los insumos de educación se encuentran en función de la nutrición de los niños en periodos previos. Esto se explica debido a que los padres actualizan sus decisiones en cada etapa con base en shocks de nutrición ocurridos en etapas previas.

Entonces, una forma reducida de la ecuación de insumos escolares adquiridos por los padres es de la siguiente manera:

$$
\begin{gathered}
R E_{1}=r e_{1}\left(Y, E M, E P, P_{E}, E, \sigma, P_{S, 1}, A_{1}, \delta, \lambda\right) \\
R E_{2}=r e_{2}\left(S_{1}, Y, E M, E P, P_{E}, E, \sigma, P_{S, 2}, A_{2}, \delta, \lambda\right) \\
R E_{3}=r e_{3}\left(S_{1}, S_{2}, Y, E M, E P, P_{E}, E, \sigma, P_{S, 3}, A_{3}, \delta, \lambda\right)
\end{gathered}
$$


Los años de escolaridad para el período 3 tienen la misma estructura que la ecuación 4:

$$
A E_{3}=a e_{3}\left(N_{1}, N_{2}, Y, E M, E P, P_{E}, E, \sigma, P_{S, 3}, A_{3}, \delta, \lambda\right)
$$

Dónde: $Y$ es el ingreso de los padres; $E M$ es la escolaridad de la madre; $E P$ es la escolaridad del padre; $P_{E}$ son los precios de artículos escolares (por simplicidad se asume que son constantes en el tiempo); $\sigma$ son las preferencias de los padres con respecto a la escolaridad de sus hijos; $P_{s t}$ son precios del cuidado de la nutrición para cada período; $\delta$ se refiere al ambiente de nutrición en cada período; At son las preferencias de los padres respecto a la nutrición de sus hijos; $\lambda$ es la salud innata del estudiante.

Al sustituir las ecuaciones de la 2 a la 5, en la ecuación 1 se obtiene:

$$
L_{3}=L\left(N_{1}, N_{2}, N_{3}, Y, E M, E P, P_{E}, E, \sigma, \gamma, P_{S, 1}, P_{S, 2}, P_{S, 3}, A_{1}, A_{2}, A_{3}, \delta, \lambda\right)
$$

La ecuación 6 representa una forma reducida de la ecuación 1, la cual contiene a la variable endógena $N_{3}$. De acuerdo con esta especificación, las habilidades académicas $\left(L_{3}\right)$ y la nutrición del niño en el tercer período $\left(N_{3}\right)$ cambian al mismo tiempo por las decisiones de los padres tomadas con base en variables exógenas. Por lo tanto, $L_{3}$ cambia como respuesta a $N_{3}$. Los precios de la alimentación y el ambiente de nutrición son establecidos por la política de salud pública del gobierno.

Con base en dichas decisiones del gobierno los padres seleccionan los recursos educativos que consumirán. Finalmente, se presentarán shocks aleatorios en la nutrición de los alumnos, tales como la incidencia de un programa de política social, con enfoque de alimentación a estudiantes. Así, $N_{3}$ está determinado por decisiones de política del gobierno, elecciones de los padres y por shocks en la nutrición. Ahora bien, la ecuación depende a su vez de otros valores, por lo cual debe contemplarse a los determinantes de nutrición de los alumnos en cada período por medio de las siguientes ecuaciones:

$$
\begin{gathered}
N_{1}=N_{1}\left(R S_{1}, A_{1}, S S_{1}, \lambda\right) \\
N_{2}=N_{2}\left(N_{1}, R S_{2}, A_{2}, S S_{2}, \lambda\right) \\
N_{3}=N_{3}\left(N_{2}, R S_{3}, A_{3}, S S_{3}, \lambda\right)
\end{gathered}
$$

Dónde: $R S_{t}$ son las adquisiciones de nutrición por parte de los padres en el período $t$; son los shocks de nutrición que ocurren en el período .

Así como la ecuación 1 es una función de producción para los logros educativos, las ecuaciones 7, 8 y 9 representan las funciones de producción de nutrición de los estudiantes. 
Estas ecuaciones muestran que la nutrición de etapas previas, las adquisiciones relacionadas con la nutrición por parte de los padres en el período actual, el ambiente de nutrición actual y la salud innata tienen un efecto directo sobre el estado de nutrición actual de los estudiantes.

Debido a que las adquisiciones de nutrición que hacen los padres para sus hijos se encuentran bajo control directo de los mismos, esta variable es endógena. Por tanto, debe desglosarse dicha variable. Dado que los padres seleccionan las adquisiciones tanto de educación como de nutrición al inicio de cada periodo, entonces, ambas son seleccionados de manera simultánea, por lo cual, las adquisiciones de nutrición se encuentran determinadas por las mismas variables que inciden en las adquisiciones de educación, tal como se presenta en las siguientes ecuaciones:

$$
\begin{aligned}
& R S_{1}=r s_{1}\left(Y, E M, E P, P_{E}, E, \sigma, P_{S, 1}, A_{1}, \delta, \lambda\right) \\
& R S_{2}=r s_{2}\left(Y, E M, E P, P_{E}, E, \sigma, P_{S, 2}, A_{2}, \delta, \lambda\right) \\
& R S_{3}=r s_{3}\left(Y, E M, E P, P_{E}, E, \sigma, P_{S, 3}, A_{3}, \delta, \lambda\right)
\end{aligned}
$$

Con base en estas ecuaciones, se puede sustituir $N_{1}, N_{2}$ y $N_{3}$, y en la ecuación 6 con sus determinantes presentados en las ecuaciones de la 7 a la 9 y entonces las adquisiciones de nutrición de estas tres ecuaciones deben también ser remplazadas por su ecuación de determinantes representado por sus ecuaciones de la 10 a la 12 , obteniendo la verdadera forma reducida de la función de calificaciones, donde todas las variables que están en la función son exógenas:

$$
L_{3}=l_{F R}\left(Y, E M, E P, P_{E}, E, \sigma, \gamma, P_{S, 1}, P_{S, 2}, P_{S, 3}, A_{1}, A_{2}, A_{3}, \delta, \lambda, S S_{1}, S S_{2}, S S_{3}\right)
$$

Esta última ecuación es una forma reducida de los determinantes de los logros escolares. La ecuación 13 tiene la característica que las variables explicativas son consideradas como exógenas, lo cual permite identificar el efecto de un programa de política pública de nutrición sobre las calificaciones de las niñas y niños. Esta especificación será usada como la base para el modelo empírico de la siguiente sección.

\section{Modelo Probit Ordenado}

Para estimar una aproximación de la ecuación 13, se utiliza una regresión típica que controla por los diferentes grupos de covariables incluidas en dicha ecuación. También se incorporan efectos fijos por escuela y por delegación. La ecuación de estimación básica está dada por la siguiente especificación. 


$$
Y_{a e d}=\alpha+\varphi \mathrm{T}_{a e d}+\tau \mathrm{X}_{a e d}+\mathrm{v}_{e}+\mathrm{e}_{d}+\mu
$$

Dónde: $Y_{\text {aed }}$ : es el resultado de logro escolar-calificaciones- para cada alumno $a$ de la escuela $e$ en la delegación $d$; es igual a 1 para todos los participantes en el programa de Desayunos Escolares; es un vector de covariables de características de los estudiantes, los hogares, las escuelas, la política social y de la comunidad; $v_{e}$ son los efectos fijos por escuela; $\mathrm{e}_{d}$ son los efectos fijos por delegación y $\mu$ : es el término de error aleatorio.

Cuando la variable que deseamos explicar no es continua, sino de rangos múltiples, la estimación por medio de modelos de Mínimos Cuadrados Ordinarios (MCO) presenta limitantes como las de todas las estimaciones de tipo binario: heterocedasticidad y probabilidades predichas superiores a la unidad (Jackman, 2000). Modelos más adecuados y ampliamente utilizados para este tipo de datos son los llamados multinomiales. Este tipo de modelos son empleados cuando la variable dependiente es categórica y toma valores discretos que, a diferencia de los modelos binomiales, contemplan varios resultados mutuamente excluyentes. Debido a que la distribución de dichos datos es de tipo multinomial, el método de estimación es por máxima verosimilitud (Cameron y Trivedi, 2005).

Específicamente, cuando la naturaleza de los datos hace que las categorías sean ordenadas, un modelo estándar para su tratamiento es el de probit ordenado. En los modelos de respuesta ordenada los valores que se asignan a cada respuesta no son arbitrarios, sino que un rango más alto representa un valor mayor. El modelo de probit ordenado puede ser derivado de un modelo de variable latente, donde es una variable de respuesta ordenada que toma valores de $\{1,2,3, \ldots . N\}$ (Wooldridge, 2002). El modelo inicial con variable latente puede expresarse como:

$$
\mathrm{y}^{*}=\mathrm{x} \beta+e, \quad e \mid x \sim \operatorname{Normal}(0,1)
$$

Dónde: $\chi$ no contempla intercepto y $\beta$ es de tamaño Jx 1 . Además, $\alpha_{1}<\alpha_{2} \ldots \alpha_{\mathrm{N}}$, representan los puntos de corte, se define que:

$$
\begin{gathered}
y=0 \text { si } y^{*} \leq \alpha_{1} \\
y=1 \text { si } \alpha_{1}<y^{*} \leq \alpha_{2} \\
\vdots \\
y=N \text { si } y^{*}>\alpha_{N}
\end{gathered}
$$

En este sentido, cuando tome valores de 1,2 y 3 existirán dos puntos de corte: $\alpha_{1}$ y $\alpha_{2}$. 
Bajo el supuesto de normalidad para $e$ es posible derivar la distribución de $\gamma$ dado $\mathrm{x}$, calculando cada probabilidad de respuesta de la forma siguiente:

$$
\begin{gathered}
P(y=0 \mid x)=P\left(y^{*} \leq \alpha_{1} \mid x\right)=P\left(x \beta+e \leq \alpha_{1} \mid x\right)=\Phi\left(\alpha_{1}+x \beta\right) \\
P(y=1 \mid x)=P\left(\alpha_{1}<y^{*} \leq \alpha_{2} \mid x\right)=\Phi\left(\alpha_{2}-x \beta\right)-\Phi\left(\alpha_{1}+x \beta\right) \\
\vdots \\
P(y=N-1 \mid x)=P\left(\alpha_{N-1}<y^{*} \leq \alpha_{N} \mid x\right)=\Phi\left(\alpha_{N}-x \beta\right)-\Phi\left(\alpha_{N-1}+x \beta\right) \\
P(y=N \mid x)=P\left(y^{*}>\alpha_{N} \mid x\right)=1-\Phi\left(\alpha_{N}-x \beta\right)
\end{gathered}
$$

La suma de las probabilidades es la unidad $\mathrm{y}<\phi$ es la distribución acumulada normal estándar. Dado que el modelo probit ordenado es la generalización del modelo probit binario, cuando $N=1$, se obtiene el modelo probit estándar:

$$
P(y=1 \mid x)=1-P(y=0 \mid x)=1-\Phi\left(\alpha_{1}-x \beta\right)=\Phi\left(x \beta-\alpha_{1}\right),
$$

Los parámetros de la regresión $\alpha$ y $\beta$ son estimados por máxima verosimilitud, donde la función para cada $i$ es:

$$
\begin{gathered}
\ell_{i}(a, \beta)=1\left[y_{i}=0\right] \log \left[\Phi\left(\alpha_{1_{1}}-x_{i} \beta\right)\right]+1\left[y_{i}=1\right] \log \left[\Phi\left(\alpha_{1_{2}}-x_{i} \beta\right)\right] \\
-\Phi\left(\alpha_{1_{1}}-x_{i} \beta\right)+\cdots+1\left[y_{i}=N\right] \log \left[1-\Phi\left(\alpha_{1_{N}}-x_{i} \beta\right)\right]
\end{gathered}
$$

Dado que en este tipo de modelos interesa la probabilidad de respuesta $\mathrm{P}(y=\mathrm{n} \mid x)$ y no $E\left(y^{*} \mid x\right)=\mathrm{X} \beta$, entonces, se calculan los efectos marginales en las probabilidades:

$$
\begin{gathered}
\frac{\partial p_{0}(x)}{\partial x_{k}}=-\beta_{i} \phi\left(\alpha_{1}-x \beta\right), \frac{\partial p_{N}(x)}{\partial x_{i}}=\beta_{i} \phi\left(\alpha_{N}-x \beta\right) \\
\frac{\partial p_{N}(x)}{\partial x_{i}}=\beta_{i}\left[\phi\left(\alpha_{N-1}-x \beta\right)-\phi\left(\alpha_{N}-x \beta\right)\right], 0<n<N
\end{gathered}
$$

\section{Resultados}

La tabla 2 incluye las estimaciones de la ecuación 14, con la cual se evalúan los resultados en el promedio académico del ciclo escolar 2017-2018 en función de la participación en el programa de Desayunos Escolares, así como de un conjunto de variables de tipo individual, familiar, escolar y de la comunidad que podrían afectar el rendimiento de los estudiantes. Las estimaciones se realizan para las 17 escuelas de la Ciudad de México que son objeto 
de estudio de este documento. En todas las especificaciones se contemplan efectos fijos por escuela y por delegación, así como precios de productos de la canasta básica.

La primera y tercera columna presentan los resultados por medio MCO para las niñas y niños de cuarto y quinto grado de primaria, respectivamente. Por su parte, las columnas dos y cuatro contienen las estimaciones por medio de modelos probit ordenado para cada grado correspondiente. Dada la naturaleza categórica de la variable dependiente, resulta una mejor modelación por medio de métodos multinomiales, tal como el probit ordenado, por lo cual se hará énfasis en estos resultados. Sin embargo, como es posible observar en dicha tabla, los resultados son robustos en dirección y magnitud bajo ambas especificaciones.

Una vez controlando por todos los posibles factores que podrían incidir en el promedio escolar, la participación en el Programa de Desayunos Escolares muestra una relación positiva, pero no significativa, sobre el puntaje académico. Los resultados de los modelos de probit ordenado señalan que el efecto podría ser de $2.3 \%$ en estudiantes de cuarto y de $57.8 \%$ en alumnos de quinto. Sin embargo, aunque la dirección del impacto es positiva, debido a que los coeficientes carecen de significancia estadística tanto en la especificación de MCO como en el probit ordenado, es posible señalar que el programa no tiene efectos colaterales que propicien un incremento relevante en las calificaciones escolares. De hecho, el programa solo tendría impactos en la nutrición de las niñas y niños participantes.

Este hallazgo es importante pues programas de nutrición similares de otros países si han tenido efectos positivos en el rendimiento escolar (Meyers et al. 1989; Vermeersch y Kremer, 2004; Belot y James, 2011; Imberman y Kugler 2014; Frisvold, 2015), probablemente por el diferente contenido nutricional de los desayunos y los años de exposición al programa. Así, se requiere un seguimiento y evaluación en el tiempo para estudiar con mayor profundidad este tipo de efectos temporales.

Adicionalmente, la tabla 2 sugiere que existen otras características de los estudiantes, de sus familias, sus escuelas y sus comunidades que podrían afectar las calificaciones de los alumnos de primaria. Comenzando por las características de los estudiantes destaca el sexo. Al respecto, son las niñas de cuarto y quinto grado quienes muestran mejores promedios, en comparación con sus congéneres varones en el mismo grado. De acuerdo con el modelo de probit ordenado, las diferencias son de $35.8 \%$ y $16.2 \%$ para cada grado respectivamente, aunque dicha relación no es estadísticamente significativa en los resultados de quinto grado. Sin embargo, de acuerdo con la evidencia empírica podrían ser las niñas quienes realizan mayores esfuerzos escolares y dediquen más tiempo a estudiar y elaborar tareas en comparación con los niños (Valle et al., 2015). Este es un aspecto en el que valdría la pena profundizar en futuras líneas de investigación. 
Resulta también de gran relevancia que el promedio del ciclo escolar anterior y el número de inasistencias explican de manera significativa el promedio actual. De hecho, los alumnos con mejores calificaciones en el ciclo 2016-2017 tienden también a obtener mejores promedios en el ciclo 2017-2018. Estudiantes con calificaciones altas en el ciclo escolar previo presentan un promedio más alto en $90.1 \%$ en cuarto grado y $95.2 \%$ en quinto grado. Mientras que, las faltas escolares si contribuyen a reducir la calificación general de los alumnos. Los estudiantes de cuarto grado que presentan al menos una falta al mes reducen su promedio en $40.6 \%$, y los de quinto lo hacen en $33.1 \%$.

Existe evidencia empírica previa de que las condiciones de los hogares afectan también los resultados académicos de las niñas y niños (Meyers et al., 1989; Frisvold, 2015). Las estimaciones de este estudio sugieren que los estudiantes de cuarto grado pertenecientes a hogares con ingresos más altos podrían ver favorecidos sus promedios escolares en $6.3 \%$. Aunque en el caso de quinto grado los coeficientes son nulos y no significativos.

La escolaridad de los padres mostró un impacto significativo sobre el desempeño académico de la población de estudio. El promedio en cuarto grado fue mejor cuando el padre de familia contaba con un nivel de licenciatura o mayor, incrementando el promedio en $42.4 \%$. Por su parte, los alumnos de quinto grado presentaron calificaciones más favorables cuando la mamá tiene bachillerato $(35.0 \%)$ y licenciatura o posgrado (39.9\%). Como señalan Hernández et al. (2006), cuando los padres tienen alta escolaridad, las expectativas que sus hijos estudien son más altas.

Este mismo indicador muestra un aumento para los estudiantes de quinto grado con familias donde la mamá y el menor viven juntos (63.9\%) y para aquellos alumnos de cuarto grado cuyo papá tiene un empleo (36.3\%). Así mismo, los resultados escolares son mejores para quienes habitan en viviendas con mayor número de focos (1.0\%). Es decir, el bienestar económico representado por la calidad de la vivienda podría ser determinante de los logros escolares de estudiantes de primaria.

Los puntajes académicos podrían verse afectados por las condiciones de la escuela a la que asisten los alumnos (Vermeersch y Kremer, 2004). Una de estas características es la experiencia de los directivos. De acuerdo con las estimaciones, esta variable podría incidir de manera positiva sobre el rendimiento académico. De manera que, cuando los profesores a cargo de los planteles escolares tienen más años de experiencia, los alumnos podrían presentar una mejora en sus calificaciones en $4.2 \%$ y $2.3 \%$ en cuarto y quinto grado, respectivamente.

Finalmente, la presencia de otros programas sociales como son el de Niños Talento y Becas Escolares de la Ciudad de México podría tener una relación a favor de la obtención de mejores calificaciones escolares. El modelo para cuarto año señala que el Programa de Niños Talento podría tener una incidencia positiva de $42.9 \%$ sobre el promedio escolar. Por su parte, los estudiantes de quinto grado muestran puntajes más favorables cuando cuentan 
con el Programa de Becas Escolares (56.3\%). Estos resultados podrían señalar que varios programas que en conjunto atienden diferentes necesidades de la población vulnerable, podrían crear sinergias positivas e incidir sobre las calificaciones escolares de estas niñas y niños.

Tabla 2

Resultados en promedio escolar por participación en el programa de Desayunos Escolares

\begin{tabular}{|c|c|c|c|c|}
\hline \multirow[b]{2}{*}{ Factores determinantes } & \multicolumn{2}{|c|}{$4^{\circ}$ Grado } & \multicolumn{2}{|c|}{$5^{\circ}$ Grado } \\
\hline & $\begin{array}{c}\mathrm{MCO} \\
(1)\end{array}$ & $\begin{array}{c}\text { Probit orde- } \\
\text { nado } \\
\text { (2) }\end{array}$ & $\begin{array}{c}\mathrm{MCO} \\
(3)\end{array}$ & $\begin{array}{c}\text { Probit ordenado } \\
\text { (4) }\end{array}$ \\
\hline \multirow{2}{*}{ Participación en Desayunos Escolares } & 0.103 & 0.023 & 0.475 & 0.578 \\
\hline & $(-0.257)$ & $(0.321)$ & $(0.312)$ & $(0.352)$ \\
\hline \multirow{2}{*}{ Sexo } & $0.270 * *$ & $0.358^{* *}$ & 0.160 & 0.162 \\
\hline & $(0.120)$ & $(0.150)$ & $(0.124)$ & $(0.140)$ \\
\hline \multirow{2}{*}{ Edad en meses } & 0.020 & 0.026 & 0.006 & 0.014 \\
\hline & $(0.015)$ & $(0.018)$ & $(0.016)$ & $(0.018)$ \\
\hline \multirow{2}{*}{ Promedio escolar anterior } & $0.737 * * *$ & $0.901 * * *$ & $0.852 * * *$ & ‘ $0.952 * * *$ \\
\hline & $(0.045)$ & $(0.072)$ & $(0.044)$ & $(0.067)$ \\
\hline \multirow{2}{*}{ Probabilidad de faltar a clases } & $-0.317 * *$ & $-0.406 * *$ & $-0.272 * *$ & $-0.331 * *$ \\
\hline & $(0.127)$ & $(0.157)$ & $(0.126)$ & $(0.142)$ \\
\hline \multirow{2}{*}{ Ingreso } & & & 0.000 & 0.000 \\
\hline & & & $(0.000)$ & $(0.000)$ \\
\hline \multirow{2}{*}{ Logaritmo del ingreso } & 0.048 & 0.063 & & \\
\hline & $(0.119)$ & $(0.146)$ & & \\
\hline \multirow{2}{*}{ Habita la madre en casa } & & & 0.489 & $0.639^{*}$ \\
\hline & & & $(0.323)$ & $(0.354)$ \\
\hline \multirow{2}{*}{ El padre trabaja } & 0.381 & 0.363 & & \\
\hline & $(0.274)$ & $(0.328)$ & & \\
\hline Nivel educativo de la madre: Prepara- & & & $0.328 * *$ & $0.350 * *$ \\
\hline toria & & & $(0.144)$ & $(0.162)$ \\
\hline Nivel educativo de la madre: Licencia- & & & $0.407 * *$ & $0.399 *$ \\
\hline tura o grado mayor & & & $(0.201)$ & $(0.228)$ \\
\hline Nivel educativo del padre: Licenciatura & 0.234 & $0.424 * *$ & & \\
\hline o grado mayor & $(0.161)$ & $(0.202)$ & & \\
\hline
\end{tabular}




\begin{tabular}{|c|c|c|c|c|}
\hline \multirow[b]{2}{*}{ Factores determinantes } & \multicolumn{2}{|c|}{$4^{\circ}$ Grado } & \multicolumn{2}{|c|}{$5^{\circ}$ Grado } \\
\hline & $\begin{array}{l}\mathrm{MCO} \\
\text { (1) }\end{array}$ & $\begin{array}{l}\text { Probit orde- } \\
\text { nado } \\
\text { (2) }\end{array}$ & $\begin{array}{c}\mathrm{MCO} \\
(3)\end{array}$ & $\begin{array}{l}\text { Probit ordenado } \\
\text { (4) }\end{array}$ \\
\hline \multirow{2}{*}{ Número de focos } & 0.016 & 0.010 & & \\
\hline & $(0.020)$ & 0.025 & & \\
\hline \multirow{2}{*}{ Relación alumnos por profesor } & 0.060 & 0.075 & & \\
\hline & $(0.040)$ & $(0.049)$ & & \\
\hline \multirow{2}{*}{ Experiencia del director } & $0.032 *$ & 0.042 & 0.016 & 0.023 \\
\hline & $(0.018)$ & $(0.022)$ & $(0.014)$ & $(0.016)$ \\
\hline \multirow{2}{*}{ Programa Niños Talento } & 0.256 & $0.429 *$ & & \\
\hline & $(0.187)$ & $(0.244)$ & & \\
\hline \multirow{2}{*}{ Programa Becas Escolares } & & & $0.493^{*}$ & $0.563^{* *}$ \\
\hline & & & $(0.251)$ & $(0.285)$ \\
\hline \multirow{2}{*}{ Ubicación } & -0.044 & 0.051 & $-0.721 * *$ & $-0.873^{* *}$ \\
\hline & $(0.360)$ & $(0.452)$ & $(0.344)$ & $(0.388)$ \\
\hline Precio de los alimentos & Sí & Sí & Sí & Sí \\
\hline Delegaciones & Sí & Sí & Sí & Sí \\
\hline Escuelas & Sí & Sí & Sí & Sí \\
\hline \multirow{2}{*}{ Constante } & -2.613 & & 4.560 & \\
\hline & $(6.492)$ & & (2.813) & \\
\hline $\mathrm{R}$ cuadrada & 0.723 & & 0.672 & \\
\hline
\end{tabular}

\section{Conclusiones}

La calidad de la nutrición de las niñas y niños durante las primeras etapas de la vida es un factor fundamental para su desarrollo físico y mental, por lo cual podría ser un determinante importante de sus logros escolares (World Food Program, 2017). Los menores en situación de pobreza son más propensos a vivir en condición de inseguridad alimentaria, y, por tanto, podrían perder las oportunidades de aprendizaje que se derivan de un adecuado consumo de nutrientes.

En la Ciudad de México, el Programa de Desayunos Escolares tiene como finalidad contribuir a la sana alimentación de la comunidad infantil en edad escolar. Aun cuando el objetivo directo del programa es la mejora en la nutrición y no en los logros cognitivos, sabemos que 
un cambio en la ingesta de nutrientes podría incidir sobre los resultados académicos, tal como lo muestra la evidencia para Estados Unidos (Frisvold, 2015; Imberman y Kugler, 2014), Kenia (Vermeersch y Kremer, 2004), Reino Unido (Belot y James, 2011), India (Afridi, 2011) y Filipinas (Glewwe et al., 2001). Dicha relación no ha sido estudiada con anterioridad para el caso de los beneficiarios del programa de desayunos escolares en la Ciudad de México.

Por lo cual, este artículo realiza una contribución a la literatura al estimar por primera ocasión los efectos de la participación en el Programa de Desayunos Escolares de la Ciudad de México, sobre las calificaciones de los estudiantes. Lo anterior mediante un enfoque contrafactual, donde fueron comparados los resultados de estudiantes beneficiarios y no beneficiarios del programa. Los datos empleados provienen del levantamiento de encuestas para la evaluación de impacto del programa, la cual permitió contar con información sobre el promedio académico y el consumo del desayuno escolar durante el periodo agosto-diciembre de 2017 para 17 escuelas de la Ciudad de México.

Las estimaciones están basadas en una ecuación de los logros escolares, donde éstos se determinan por la nutrición, así como por otros factores de tipo individual, familiar, escolar y de la comunidad. Por medio de modelos de regresión lineal y de probit ordenado se encontró que la participación en el programa no presentó una relación estadísticamente significativa con los promedios alcanzados por los estudiantes. Aunque, los coeficientes son positivos (2.3\% y $57.8 \%$ en cuarto y quinto grado respectivamente) la relación es no significativa, por lo cual es posible concluir que la ingesta de los alimentos como parte del programa no propicia una diferencia importante sobre las calificaciones escolares de los estudiantes.

Otros hallazgos relevantes del estudio fueron la importancia de factores como el sexo, las faltas a clases, la escolaridad de los padres y la disponibilidad de programas sociales adicionales en la determinación del promedio escolar. Al respecto, los resultados de las estimaciones señalan que las niñas son quienes presentaron promedios de mayor rango en comparación con los niños. Así mismo, aquellos con faltas escolares disminuyeron sus puntajes escolares. Estudiantes con madres y padres más preparados en términos de nivel de escolaridad obtuvieron resultados más favorables en sus calificaciones. Finalmente, la presencia de programas sociales adicionales resultó también positiva para los puntajes académicos de estas niñas y niños.

El hecho de que el programa de Desayunos Escolares hubiera tenido mejoras sobre las variables de nutrición y calificaciones escolares implicaría que el programa de política pública tendría un efecto directo en la nutrición y un efecto colateral positivo sobre los logros escolares. Lo anterior, coadyuvaría a la reducción de las altas tasas de sobrepeso y obesidad infantil, un problema de salud nacional. Además, contribuiría a reducir las brechas de educación entre quienes tienen acceso a una mejor alimentación y quienes, por motivos de vulnerabilidad social y de ingresos, no acceden a una adecuada nutrición. Finalmente, contribuiría a la formación de capital humano, indispensable para el crecimiento económico de largo plazo de un país. 
Los resultados de la investigación abren además el panorama para futuros estudios sobre las sinergias entre los programas sociales de la Ciudad de México, de manera que sería pertinente cuestionarnos si en conjunto las estrategias que atienden a niñas y niños contribuyen positivamente a la formación de capital humano. Adicionalmente, sería importante explorar los efectos del programa de Desayunos Escolares sobre indicadores de la calidad nutricional, tales como los niveles de ingesta de vitaminas y minerales.

\section{Referencias}

Afridi, F. (2011) The impact of School Meals on School Participation: Evidence from Rural India. Journal of Development Studies. 47, (11). https://doi.org/10.1080/00220388.2010.514330

Anzman-Frasca, S., Djang, H. C., Halmo, M. M., Dolan, P. R. \& Economos, C. D (2015). Estimating Impacts of a Breakfast in the Classroom Program on School Outcomes. JAMA Pediartr 169 (1), 71-77. https://doi. org/10.1001/jamapediatrics.2014.2042

Behrman, J. R. y J. Hoddinott. (2001). An Evaluation of Impact of Progresa on Preschool Child Height. Food Consumption and Nutrition Division, FCND Discussion paper No.104. International Food Policy Research Institute, Washington, D.C.

Belot, M. \& James, J. (2011). Healthy school meals and educational outcomes. Journal of Health Economics 30 (3), 489-504. https://doi.org/10.1016/j.jhealeco.2011.02.003

Cameron, C. A \& Trivedi P. K. (2005) Microeconometrics: Methods and applications (1 ${ }^{\text {st }}$ Ed.) New York: Cambridge University Press.

Federal Interagency Forum on Child \& Family Statistics (2007). America's Children: Key National Indicators of Well-Being, 2007. U.S. Government Printing Office, Washington, DC. Disponible en: https://www.childstats. gov/pdf/ac2007/ac_07.pdf. Consultado: 28/07/2018.

Frisvold, D. E. (2015). Nutrition and cognitive achievement: An Evaluation of the School Breakfast Program Journal of Public Economics 124, 91-104. http://dx.doi.org/10.1016/j.jpubeco.2014.12.003

Gaceta oficial de la Ciudad de México (2016). Gaceta oficial de la Ciudad de México Órgano de Difusión de la Ciudad de México. No. 24. Disponible en: http://data.consejeria.cdmx.gob.mx/portal_old/uploads/gacetas/ 79c4a8f6f47333c02de3eceb04ecbf9b.pdf. Consultado: 10/04/2018.

Gaceta oficial de la Ciudad de México (2018). Gaceta oficial de la Ciudad de México Órgano de Difusión de la Ciudad de México. No. 252(tomo III). Disponible en: http://data.consejeria.cdmx.gob.mx/portal_old/uploads/ gacetas/debcb2f97537fafe489c77c1fdf2b041.pdf. Consultado: 10/04/2020.

Glewwe, P., Jacoby, H. \& King, E. (2001) Early childhood nutrition and academic achievement: a longitudinal analysis. Journal of Public Economics. 81, (3). 345-368. https://doi.org/10.1016/S0047-2727(00)00118-3

Glewwe, P. (2005). The impact of health and nutrition on education in developing countries: Theory, econometrics issues, and recent empirical evidence. Food and Nutrition Bulletin 26(2). S235-S250. https://doi.or$\mathrm{g} / 10.1177 / 15648265050262 \mathrm{~S} 215$

Henández J., Márquez A. \& Palomar J. (2006). Factores asociados con el desempeño en el EXANI-I Zona Metropolitana de la Ciudad de México 1996-2000. Revista Mexicana de Investigación Educativa 11 (29), pp. 547-581. Disponible en: http://www.scielo.org.mx/scielo.php?script=sci_arttext\&pid=S1405-66662006000200547\&ln$\mathrm{g}=$ es\&nrm=iso. Consultado: 11/06/2018.

Hinrichs, P. (2010). The Effects of the National School Lunch Program on Education and Health. Journal of Policy Analysis and Management. 27 (3). 479-505. https://doi.org/10.1002/pam.20506 
Imberman, S. A. \& Kugler, A. D. (2014). The Effect of Providing Breakfast in Class on Student Performance. Journal of Policy Analysis and Management 33(3), 669-699. https://doi.org/10.1002/pam.21759

Jackam, S. (2000). Models for Ordered Outcomes. Political Science 200C. Disponible en: https://web.stanford.edu/ class/polisci203/ordered.pdf. Consultado: 21/07/2018.

McEwan, P. J. (2013). The impact of Chile's school feeding program on education outcomes. Economics of Education Review 32, 122-139. https://doi.org/10.1016/j.econedurev.2012.08.006

Meyers, A. F., Sampson, A. E., Weitzman, M., Rogers, B. L. \& Kayne, H. (1989). School Breakfast Program and School Performance. American journal of diseases of children 143(10),1234-1239. https://doi.org/10.1001/ archpedi.1989.02150220142035.

Sistema Nacional para el Desarrollo Integral de Familia y Dirección General de Alimentación y Desarrollo Comunitario (2017). Lineamientos de la Estrategia Integral de Asistencia Social Alimentaria 2017. Secretaría de Salud, DIF. Disponible en: http://sitios.dif.gob.mx/dgadc/wp-content/uploads/2017/01/Lineamientos-EIASA-20171.pdf. Consultado: 18/04/2018.

Torres, F. \& Rojas, A. (2018). Obesidad y salud pública en México: Transformación del patrón hegemónico de oferta-demanda de alimentos. Revista Problemas del Desarrollo 193 (49). http://dx.doi.org/10.22201/ iiec.20078951e.2018.193.63185

Valle A., Pan I., Núñez J.C., Rosário P. Rodríguez S. \& Regueiro B. (2015). Deberes escolares y rendimiento académico en Educación Primaria. Anales de Psicología 31(2), pp. 562-659. http://dx.doi.org/10.6018/analesps.31.2.171131

Vermeersch, C., y M. Kremer. (2005). School Meals, Educational Achievement and School Competition: Evidence from a Randomized Evaluation. Policy Research Working Paper; No. 3523. World Bank, Washington, DC. Disponible en: https://openknowledge.worldbank.org/handle/10986/8884. Consultado: 03/05/2018.

Wooldridge, J. (2002). Econometric Analysis of Cross Section and Panel Data (1 $1^{\text {st }}$ Ed.) Masschusetts, Cambridge.

World Food Programme (2017). Smart School Meals, Nutrition-Sensitive National Programmes in Latin America and the Caribbean, A Review of 16 Countries. Disponible en: https://docs.wfp.org/api/documents/WFP0000019946/download/. Consultado: 05/06/2018. 